\title{
The mPIVAS study:
}

\section{efficacy of PsyLOG application in monitoring of antipsychotics' side-effects}

\author{
Dina Bosnjak Kuharic ${ }^{1}$, Porin Makaric ${ }^{1}$, Ante Silic ${ }^{2}$, Drazenka Ostojic ${ }^{1}$, Ilaria Culo ${ }^{1}$, Aleksandar Savic ${ }^{1}$, \\ Natasa Duran ${ }^{1}$, Zarko Bajic ${ }^{3}$, Mirela Celic Ruzic ${ }^{3}$, Daniela Petric ${ }^{4}$, Martina Rojnic Kuzman ${ }^{5}$ \\ ${ }^{1}$ University Psychiatric Hospital Vrapce, Zagreb, Croatia; ${ }^{2}$ Department of Psychiatry, Sestre milosrdnice University Hospital Center, \\ Zagreb, Croatia; ${ }^{3}$ Psychiatric Hospital Sveti Ivan, Zagreb Croatia; ${ }^{4}$ Department of Psychiatry, Rijeka University Hospital Centre, \\ Rijeka, Croatia; ${ }^{5}$ Department of Psychiatry, Zagreb University Hospital Centre, Zagreb, Croatia
}

Presented at The 19th WPA World Congress of Psychiatry, Lisbon, Portugal, 21-24 August 2019

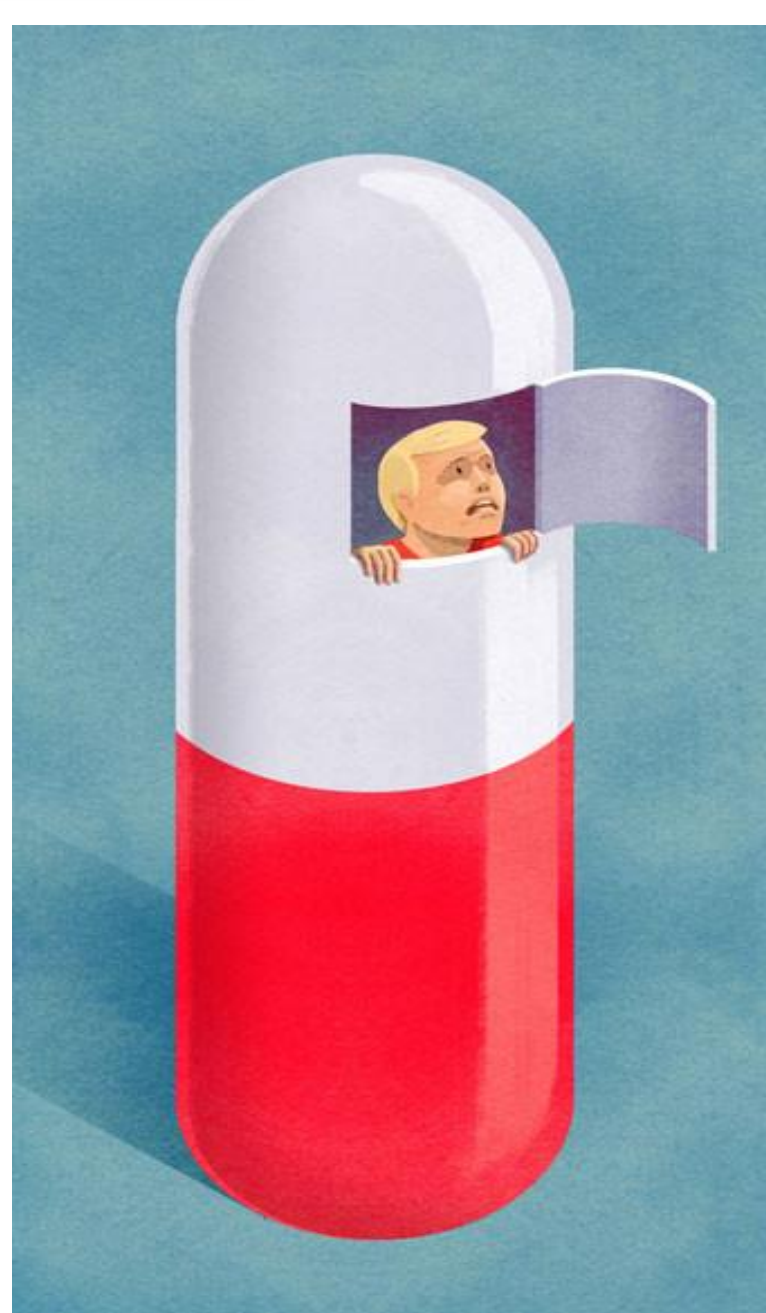

\section{Background}

While being very potent therapeutic agents, antipsychotics are frequently associated with a number of side effects that contribute to a reduced quality of life and secondary medical complications in patients. Monitoring of side-effects of antipsychotics (SEA) is often subjective, depending on ad-hoc reporting from patients and their care-givers. It frequently leads to prolonged duration of SEA, possible therapy discontinuation and finally to relapse of mental illness. Therefore, adequate detection of SEA is crucial for the patients' health.

\section{mPIVAS study and PsyLOG application}

The first mPIVAS study compared users $(n=36)$ and non-users $(n=42)$ of Android operating system mobile application for tracking SEA, PsyLOG. The PsyLOG consists of several categories including 'My Drug Effects' category with a list of 30 side effects and the possibility to add additional three. Each side effect has an appropriate description and its severity can be rated on a visual analogue scale from 0 to $100 \%$. Results showed that in contrast to standardized psychiatric assessments tools, using PsyLOG application enabled patients to report more SEA. Also, SEA were reported soon after they occured with a median (interquartile range) of 5 (3-9.5) days after the last assessment point.

\section{Protocol for the new mPIVAS study}

Aim:

To test the use of mobile technology for improvement of SEA monitoring.

Study design:

Randomized control trial.

Participants:

Patients diagnosed with schizophrenia spectrum disorder.

Antipsychotic treatment for up to 5 years.

Recruitment plan:

195 (80\% power with expected $\leq 15 \%$ of drop out and missing data).

\section{Methods:}

Randomization to one of the 3 groups:

\section{PsyLOG application} $(n=65)$

\section{GASS questionnaire $(n=65)$}

Standard monitoring $(n=65)$
Baseline

Additional assessment: 1 month Quality of life Therapeutic alliance
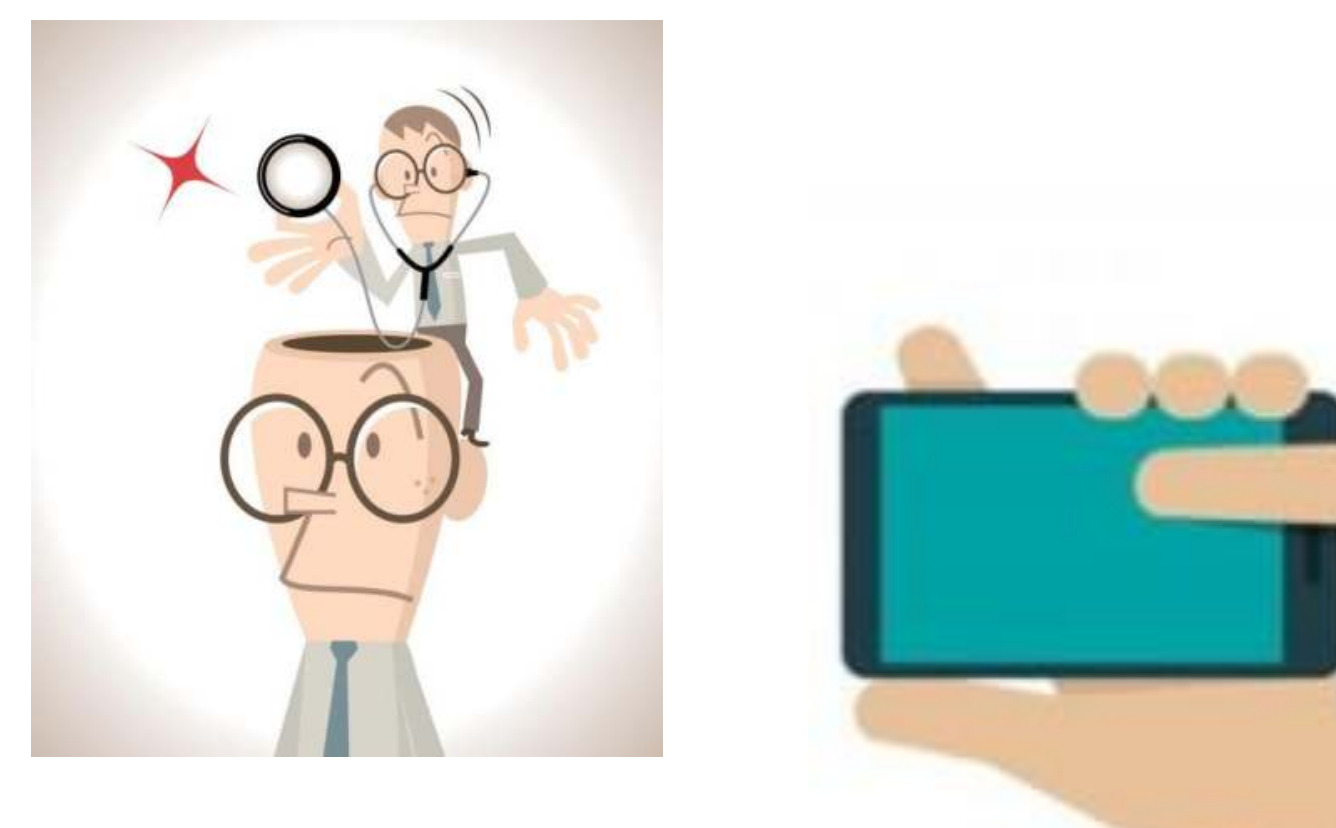

Analysis of:

- differences between 3 groups in the proportion of patients whose treatment was modified due to SEA and the median time for it

- changes in therapeutic alliance 3 months and patients' quality of life

\section{Conclusions:}

Identifying effective measures for monitoring of SEA could contribute to patients' health, quality of life and adherence by improving their knowledge about pharmacotherapy, recognition of side-effects and involvement in their treatment, as well as to help clinicians in choosing more appropriate medications. Additionally, as mental health services frequently allow no more than one consultation per month for out-patients, time between reporting side effects and their treatment might be considerably too long. The use of PsyLOG may shorten the time of recording the side effects and possibly allow contact with the therapist on time.

\section{References:}

1. Rojnic Kuzman M, Andlauer O, Burmeister K, Dvoracek B, Lencer R, Koelkebeck K, Nawka A, Riese F. The PsyLOG mobile application: development of a tool for the assessment and monitoring of side effects of psychotropic medication. Psychiatr Danub. 2017;29(2):214-217.

2. Rojnic Kuzman M, Andlauer O, Burmeister K, Dvoracek B, Lencer R, Koelkebeck K, Maric NP, Nawka A, Pantovic-Stefanovic M, Riese F, Aukst Margetic B, Bosnjak D, Ruzic MC, Curkovic M, Grubsin J, Madzarac Z, Makaric P, Petric D, Radic K, Savic A. Effective assessment of psychotropic medication side effects using PsyLOG mobile application. Schizophr Res. 2018;192:211-212. 